Research Article

\title{
A Novel Description on Vague Graph with Application in Transportation Systems
}

\author{
Zheng Kou $\mathbb{D}^{1},{ }^{1}$ Saeed Kosari $\mathbb{D},{ }^{1}$ and Maryam Akhoundi $\mathbb{D}^{2}$ \\ ${ }^{1}$ Institute of Computing Science and Technology, Guangzhou University, Guangzhou 510 006, China \\ ${ }^{2}$ Clinical Research Development Unit of Rouhani Hospital, Babol Univetsity of Medical Sciences, Babol, Iran \\ Correspondence should be addressed to Saeed Kosari; saeedkosari38@gzhu.edu.cn
}

Received 14 October 2021; Revised 11 November 2021; Accepted 16 November 2021; Published 2 December 2021

Academic Editor: Lazim Abdullah

Copyright (c) 2021 Zheng Kou et al. This is an open access article distributed under the Creative Commons Attribution License, which permits unrestricted use, distribution, and reproduction in any medium, provided the original work is properly cited.

Fuzzy graph (FG) models embrace the ubiquity of existing in natural and man-made structures, specifically dynamic processes in physical, biological, and social systems. It is exceedingly difficult for an expert to model those problems based on a FG because of the inconsistent and indeterminate information inherent in real-life problems being often uncertain. Vague graph (VG) can deal with the uncertainty associated with the inconsistent and determinate information of any real-world problem, where FGs many fail to reveal satisfactory results. Regularity definitions have been of high significance in the network heterogeneity study, which have implications in networks found across biology, ecology, and economy; so, adjacency sequence (AS) and fundamental sequences (FS) of regular vague graphs (RVGs) are defined with examples. One essential and adequate prerequisite has been ascribed to a VG with maximum four vertices is that it should be regular based on the adjacency sequences concept. Likewise, it is described that if $\zeta$ and its principal crisp graph (CG) are regular, then all the nodes do not have to have the similar AS. In the following, we obtain a characterization of vague detour (VD) g-eccentric node, and the concepts of vague detour g-boundary nodes and vague detour g-interior nodes in a VG are examined. Finally, an application of vague detour g-distance in transportation systems is given.

\section{Introduction}

The graph concept stands as one of the most dominant and widely employed tools for the multiple real-world problem representation, modeling, and analyses. To represent the objects and the relations between them, the graph vertices and edges are applied, respectively. FG-models are beneficial mathematical tools for addressing the combinatorial problems in various fields involving research, optimization, algebra, computing, environmental science, and topology. Thanks to the natural existence of vagueness and ambiguity, fuzzy graphical models are strikingly better than graphical models. Rosenfeld [1] proposed the idea of FG in 1975. Akin to the set theory, the historical past of the FG theory is the fuzzy set theory developed by Zadeh [2] in 1965. The notion of vague set theory, the generalization of Zadeh's fuzzy set theory, was introduced by Gau and Buehrer [3] in 1993. The VSs describe more possibilities than fuzzy sets. VS is more effective for the existence of the false membership degree. Kauffman [4] represented FGs based on Zadeh's fuzzy relation [5, 6]. Akram et al. $[7,8]$ described several concepts and results of FGs. Samanta et al. $[9,10]$ represented fuzzy competition graphs and some remarks on bipolar fuzzy graphs. Gani et al. [11, 12] investigated on irregular and regular fuzzy graphs. Sunitha et al. $[13,14]$ studied complement of a fuzzy graph. VG notion was introduced by Ramakrishna in [15]. Borzooei et al. [16, 17] investigated new concepts of VGs. Gary Chartrand [18] discussed the concepts of detour center of a graph. The notion of detour number, detour set, detour nodes, and detour basis in a graph were established by Gary Chatrand, G. L. Johns, and P. Zhang [19]. Interior nodes and boundary nodes are discussed by G. Chatrand, D. Erwin, G. L. Johns, and P. Zhang [20]. Fuzzy detour g-distance was given by J. P. Linda and M. S. Sunitha [21]. Ghorai et al. [22, 23] defined detour g-interior nodes in bipolar fuzzy graphs and characterization of regular bipolar fuzzy graphs. The idea of strong arcs in FG was given by Bhutani and Rosenfeld [24], and types of arc in FG were given by Mathew and Sunitha [25]. The notion of bridge, trees, cycles, 
and end nodes were described by Rosenfeld [1]. Rosenfeld and Bhutani [24] represented the notion of g-distance in FG. Also, the notions of g-boundary node, g-interior node, and g-eccentric node were defined by Linda and Sunitha [26].

A VG is referred to as a generalized structure of an FG that conveys more exactness, adaptability, and compatibility to a system when coordinated with systems running on FGs. Also, a VG is able to concentrate on determining the uncertainly coupled with the inconsistent and indeterminate information of any real-world problem, where FGs may not lead to adequate results.

The concepts of regularity play an important part in both graph theory and application in the vague environment. The highly regular graphs characterization has also been applied to the question of heterogeneity, yet all of these fail to shed enough light on real-world situations; hence, in this paper, AS and FS of RVGs are defined with examples. One essential and adequate prerequisite has been ascribed to a VG with maximum four vertices is that it should be regular based on the AS concept. Detection of nodes on the network boundary is necessary for correct operation in many wireless applications. Also, nodes close to network boundary are often assumed to provide the best candidate for beacon nodes in virtual co-ordinate construction. Focusing on these applications, a research is carried out for boundary nodes and interior ones in vague graph. A characterization of vague detour g-eccentric node and the concepts of vague detour g-boundary nodes and vague detour g-interior nodes in a VG are examined. Finally, an application of vague detour g-distance in transportation systems is given. Recently, some research has been conducted by the authors in the continuation of previous works related to VGs, bipolar fuzzy graphs, and intuitionistic fuzzy graphs which are mentioned in [27-38]. Shoaib et al. [39] introduced new concepts of pythagorean fuzzy graphs.

\section{Preliminaries}

A graph denotes a pair $G^{*}=(V, E)$ satisfying $E \subseteq V \times V$. The elements of $V$ and $E$ are the nodes and edges of the graph $G^{*}$, correspondingly. An FG has the form of $G=(\lambda, \nu)$, where $\lambda: V \longrightarrow[0,1]$ and $v: V \times V \longrightarrow[0,1]$ as is defined by $\nu(a b) \leq \lambda(a) \wedge \lambda(b)$ and $\forall a, b \in V$ and $\nu$ is a symmetric fuzzy relation on $\lambda$ and $\wedge$ denotes the minimum.

The FS of an FG $G=(\lambda, \nu)([40])$ is described as follows:

$$
f_{S}(G)=\{\lambda(a)>0, a \in V\} \cup\{\nu(a, b)>0, a, b \in V\},
$$

in which the elements are taken from the interval $[0,1]$.

Definition 1 (see [3]). A VS $M$ is a pair $\left(t_{M}, f_{M}\right)$ on set $V$ that $t_{M}$ and $f_{M}$ are real-valued functions which can be defined on $V \longrightarrow[0,1]$, so that $t_{M}(a)+f_{M}(a) \leq 1, \forall a \in V$.

Definition 2 (see [15]). A pair $\zeta=(M, N)$ is called to be a VG on a CG $G^{*}$, where $M=\left(t_{M}, f_{M}\right)$ is a VS on $V$ and $N=\left(t_{N}, f_{N}\right)$ is a VS on $E \subseteq V \times V$ so that $t_{M}(a b) \leq$ $\min \left(t_{M}(a), t_{M}<(b)\right)$ and $f_{N}(a b) \geq \max \left(f_{M}(a), f_{M}(b)\right)$, for every edge $a b \in E$.
Definition 3 (see [16]). The complement of a VG $\zeta$ is a VG $\bar{\zeta}=(\bar{M}, \bar{N})$, where $\bar{M}=M$ and $\bar{N}=\left(\overline{t_{N}}, \overline{f_{N}}\right)$ are defined by the following:

$$
\begin{aligned}
& \overline{t_{N}}(a b)=\min \left(t_{M}(a), t_{M}(b)\right)-t_{N}(a b), \\
& \overline{f_{N}}(a b)=f_{N}(a b)-\max \left(f_{M}(a), f_{M}(b)\right), \quad \text { for all } a b \in E .
\end{aligned}
$$

Definition 4 (see [17]). Let $\zeta=(M, N)$ be a VG.

(i) Then, OND of $a \in V$ is defined by $\operatorname{deg}(a)=$ $\left(\operatorname{deg}^{t}(a), \operatorname{deg}^{f}(a)\right)$, that $\operatorname{deg}^{t}(a)=\sum_{a \neq b} t_{N}(a b)$ and $\operatorname{deg}^{f}(a)=\sum_{\substack{a \neq b \\ a b \in E}} f_{N}(a b)$. If all the nodes of $\zeta$ have the same OND $\left(m_{1}, m_{2}\right)$, then, $\zeta$ is called to be $\left(m_{1}, m_{2}\right)$-regular.

(ii) The CND of $a \in V$ in $\zeta$ is denoted as $\operatorname{deg}[a]=$ $\left(\operatorname{deg}^{t}[a], \operatorname{deg}^{f}[a]\right)$, where $\operatorname{deg}^{t}[a]=\operatorname{deg}^{t}(a)+t_{M}(a)$ and $\operatorname{deg}^{f}[a]=\operatorname{deg}^{f}(a)+f_{M}(a)$. If every node of $\zeta$ has equal closed neighborhood degree $\left(n_{1}, n_{2}\right)$, then $\zeta$ is called to be $\left(n_{1}, n_{2}\right)$-totally regular.

Definition 5 (see [17]). Let $\zeta$ be a VG. If $t_{N}(a b)=$ $\min \left\{t_{M}(a), t_{M}(b)\right\}$ and $f_{N}(a b)=\max \left\{f_{M}(a), f_{M}(b)\right\}$, $\forall a, b \in V$, then $\zeta$ is called a complete-VG and if $t_{N}(a b)=$ $\min \left\{t_{M}(a), t_{M}(b)\right\}$ and $f_{N}(a b)=\max \left\{f_{M}(a), f_{M}(b)\right\}$, $\forall a b \in E$, then, $\zeta$ is called a strong-VG of the CG $G^{*}=(V, E)$.

Definition 6 (see [7]). Let $L\left(G^{*}\right)=(Y, Z)$ be a line graph of a CG $G^{*}$. Suppose $\zeta=(M, N)$ be a VG of $G^{*}$. Then, a vague line graph $L(\zeta)=\left(M_{1}, N_{1}\right)$ of $\zeta$ is described as follows:

(i) $M_{1}$ and $N_{1}$ are vague subsets of $Y$ and $Z$, respectively

$$
\begin{aligned}
& \text { (ii) } t_{M_{1}}\left(R_{m}\right)=t_{N}(m)=t_{N}\left(u_{m} v_{m}\right) \\
& \text { (iii) } f_{M_{1}}\left(R_{m}\right)=f_{N}(m)=f_{N}\left(u_{m} v_{m}\right) \\
& \text { (iv) } t_{N_{1}}\left(R_{m} R_{n}\right)=\min \left\{t_{N}(m), t_{N}(n)\right\}=\min \left\{t_{N}\left(u_{m} v_{m}\right),\right. \\
& \left.t_{N}\left(u_{n} v_{n}\right)\right\} \\
& \left(\text { v) } f_{N_{1}}\left(R_{m} R_{n}\right)=\max \left\{f_{N}(m), f_{N}(n)\right\}=\max \left\{f_{N}\right.\right. \\
& \left.\left(u_{m} v_{m}\right), f_{N}\left(u_{n} v_{n}\right)\right\}, \forall R_{m}, R_{n} \in Y \text { and } R_{m} R_{n} \in Z
\end{aligned}
$$

Definition 7 (see [16]). Let $\zeta$ be a VG and $a, b \in V$.

(i) A path P: $a=a_{0}, a_{1}, \ldots, a_{k}=b$ in $\zeta$ is a sequence of distinct nodes so that $t_{N}\left(a_{i-1} a_{i}\right)>0, f_{N}\left(a_{i-1} a_{i}\right)>0$, $i=1,2, \ldots, k$ and the length of the path is $k$.

(ii) If $P$ : $a=a_{0}, a_{1}, \ldots, a_{k}=b$ be a path of length $k$ between $a$ and $b$, then, $\left(t_{N}(a b)\right)^{k}$ and $\left(f_{N}(a b)\right)^{k}$ are defined as $\left(t_{N}(a b)\right)^{k}=\sup \left\{t_{N}\left(a a_{1}\right) \wedge t_{N}\left(a_{1} a_{2}\right) \wedge \cdots \wedge\right.$ $\left.t_{N}\left(a_{k-1} b\right)\right\} \quad$ and $\left(f_{N}(a b)\right)^{k}=\inf \left\{f_{N} \quad\left(a a_{1}\right) \vee\right.$ $\left.f_{N}\left(a_{1} a_{2}\right) \vee \cdots \vee f_{N}\left(a_{k-1} b\right)\right\} . \quad\left(t_{N}^{\infty}(a b), f_{N}^{\infty}(a b)\right)$ is said to be the strength of connectedness between two nodes $a$ and $b$ in $\zeta$, where $t_{N}^{\infty}(a b)=\sup \left(t_{N}^{k}(a b)\right)$ and $f_{N}^{\infty}(a b)=\inf \left(f_{N}^{k}(a b)\right)$. If $t_{N}(a b) \geq t_{N}^{\infty}(a b)$ and $f_{N}(a b) \leq f_{N}^{\infty}(a b)$, then, the arc $a b$ is said to be a 
strong arc. A path $a-b$ is strong path if all arcs on the path are strong.

Definition 8 (see [16]). A connected-VG $\zeta$ is said to be a vague tree if $\zeta$ has a vague spanning subgraph $S=(A, E)$ which is also a vague tree and for all $\operatorname{arcs}(a, b)$ not in $S$, $t_{N}(a b)<t_{N}^{\infty}(a b)$ and $f_{N}(a b)>f_{N}^{\infty}(a b)$. The vague spanning subgraph $S$ of $\zeta$ is a maximum spanning subgraph of $\zeta$ if $\zeta$ has no vague spanning subgraph different from $S$ contains $S$.

Notations are shown in Table 1.

\section{New Concepts in Regular Vague Graphs}

Definition 9. The AS of a node $a$ in a VG $\zeta$ is described by $A S(a)=\left(A S(a)_{t}, A S(a)_{f}\right)$, where $A S(a)_{t}=\left(t_{1}, t_{2}, \ldots, t_{n}\right)$ and $A S(a)_{f}=\left(f_{1}, f_{2}, \ldots, f_{n}\right)$ signify the sequence of TMVs and FMVs of the edges neighbor to a arranged in ascending order, respectively.

Example 1. Consider the VG $\zeta$ as Figure 1. The AS of the nodes are

$$
\begin{aligned}
& A S(a)=\left(A S(a)_{t}, A S(a)_{f}\right)=((0.1,0.2),(0.5,0.7)), \\
& A S(b)=\left(A S(b)_{t}, A S(b)_{f}\right)=((0.1,0.2,0.2),(0.5,0.6,0.6)), \\
& A S(c)=\left(A S(c)_{t}, A S(c)_{f}\right)=((0.1,0.1),(0.6,0.7)), \\
& A S(d)=\left(A S(d)_{t}, A S(d)_{f}\right)=((0.1,0.2,0.2),(0.6,0.7,0.8)), \\
& A S(e)=\left(A S(e)_{t}, A S(e)_{f}\right)=((0.1,0.2),(0.7,0.8)) .
\end{aligned}
$$

\section{Remark 1}

(i) The elements number in $\operatorname{AS}(a)_{t}$ or $\operatorname{AS}(a)_{f}$ is the degree of $a$ in $G^{*}$.

(ii) The sum of all elements in $\operatorname{AS}(a)_{t}$ and the sum of all elements in $\operatorname{AS}(a)_{f}$ is $\operatorname{deg}(a)$ in $\operatorname{VG} \zeta$, i.e.,

$$
\operatorname{deg}(a)=\left(\sum_{t_{i} \in A S(a)_{t}} t_{i}, \sum_{t_{i} \in A S(a)_{f}} f_{i}\right)
$$

Remark 2. If $\zeta$ is a RVG, then $G^{*}$ does not have to be regular and all the nodes do not need to have the same AS.

Now, we present an example that proves the correctness of the above remark.

Example 2. Consider the VG $\zeta$ in Figure 2.

In this example, $\zeta$ is a $(0.4,0.8)-\mathrm{RVG}$, but $G^{*}$ is not regular and the nodes in $\zeta$ do not share the same AS.

$$
\begin{aligned}
\operatorname{AS}(b) & =((0.1,0.1,0.2),(0.2,0.2,0.4)) \neq \operatorname{AS}(a) \\
& =((0.2,0.2),(0.4,0.4)) .
\end{aligned}
$$

TABLE 1: Some basic notations.

\begin{tabular}{lc}
\hline Notation & Meaning \\
\hline FG & Fuzzy graph \\
VS & Vague set \\
VG & Vague graph \\
AS & Adjacency sequence \\
FS & Fundamental sequence \\
RVG & Regular vague graph \\
CG & Crisp graph \\
OND & Open neighborhood degree \\
CND & Closed neighborhood degree \\
TMV & True membership value \\
FMV & False membership value \\
FFS & First fundamental sequence \\
SFS & Second fundamental sequence \\
VLG & Vague line graph \\
CVG & Connected vague graph \\
RG & Regular graph \\
MV & Membership value \\
VEN & Vague end node \\
VD & Vague detour \\
\hline
\end{tabular}

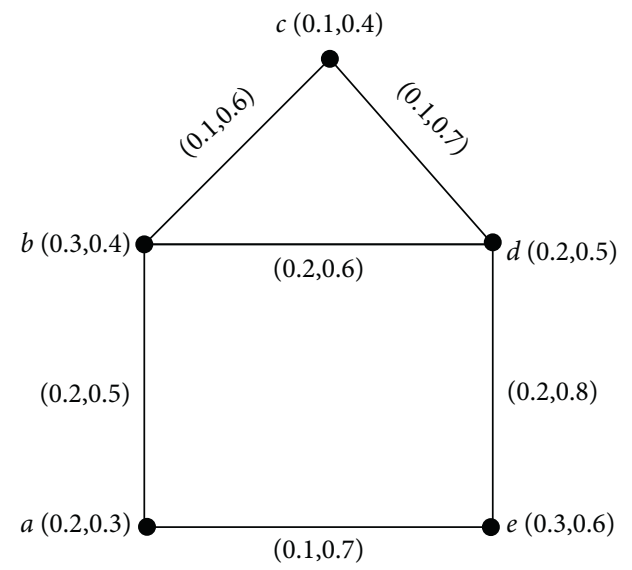

FIgURE 1: Vague graph $\zeta$ and its AS.

Hence, in a RVG, all the nodes do not need to have the similar AS.

Remark 3. If $\zeta$ and $\zeta^{*}$ are RGs, then all the nodes do not need to have the similar AS.

Example 3. Consider the VG $\zeta$ as Figure 3.

Here, $\quad \operatorname{deg}(a)=\operatorname{deg}(b)=\operatorname{deg}(c)=\operatorname{deg}(d)=\operatorname{deg}(e)=$ $(0.4,1.5)$. So, $\zeta$ is a RVG. Correspondingly, we observe that $G^{*}$ is regular. But ads $(a)=((0.1,0.1,0.2)$, $(0.4,0.5,0.6)) \neq \operatorname{ads}(f)=((0.1,0.1,0.2),(0.4,0.4,0.7))$.

Definition 10. The FS of a VG $\zeta$ is defined as $f_{s}(\zeta)=\left(F_{s^{t}}(\zeta), f_{s^{f}}(\zeta)\right)$, where

$$
\begin{aligned}
& f_{s^{t}}(\zeta)=\left\{t_{M}(a)>0 ; a \in V\right\} \cup\left\{t_{N}(a b)>0: a, b \in V\right\}, \\
& f_{s^{f}}(\zeta)=\left\{f_{M}(a)>0 ; a \in V\right\} \cup\left\{f_{N}(a b)>0: a, b \in V\right\} .
\end{aligned}
$$




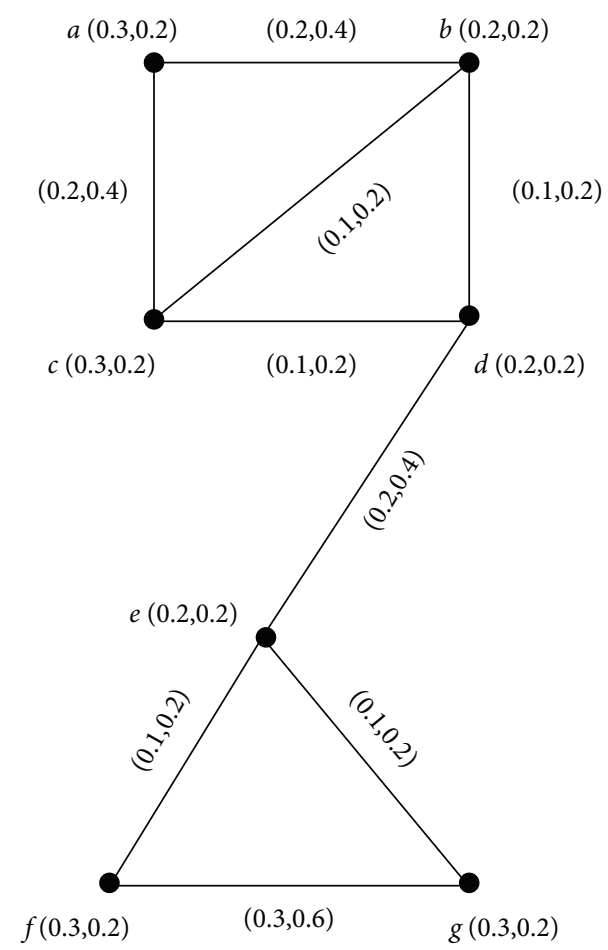

Figure 2: $\operatorname{Rvg} \zeta$.

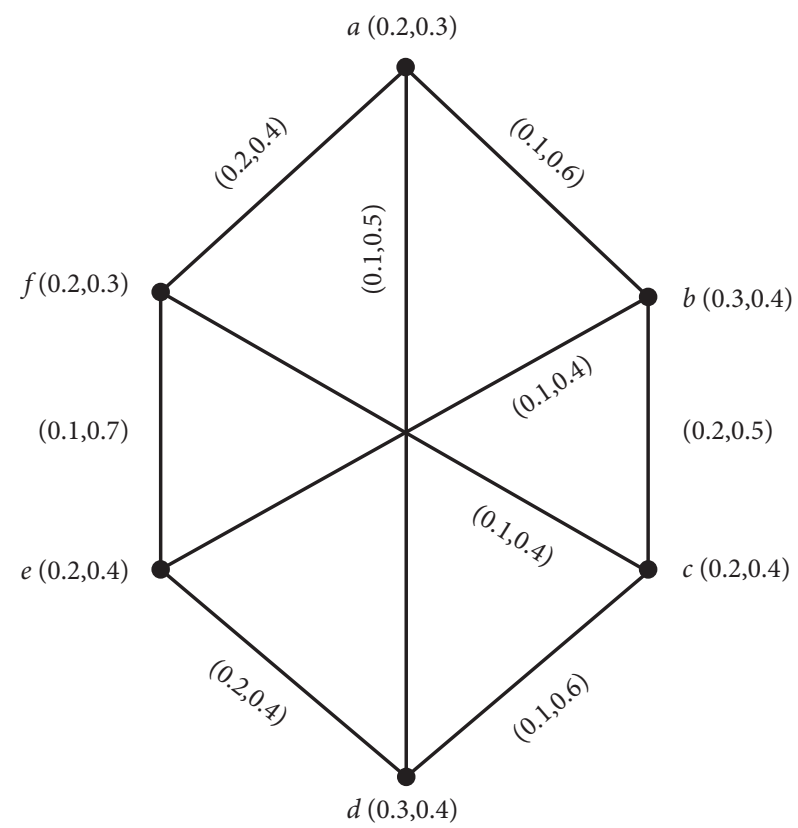

Figure 3: Regular graphs $\zeta$ and $G^{*}$ with different adjacency sequence.

The elements of $f_{s^{t}}(\zeta)$ and $f_{s^{f}}(\zeta)$ are ordered in descending and ascending order, respectively. The node part of the FS is characterized as the FFS and the edge part of the FS is labeled as the SFS and described by $f f s(\zeta)$ and $s f s(\zeta)$, respectively.

Theorem 1. Let $\zeta$ be a VG. If $n \leq 4$, then $\zeta$ is a $R V G$ iff $G^{*}$, i.e., $R G$ and all the nodes have the similar AS, $n$ being the number of nodes in $\zeta$.
Proof. Suppose $G^{*}$ is a RG and all the nodes have the similar AS. Then, $\zeta$ is a RVG.

On the contrary, let $\zeta$ be a $d=\left(d_{1}, d_{2}\right)$-RVG. If there is no edge among the nodes or if the number of nodes is one, then there is nothing to prove. Thus, we suppose that $E \neq \varnothing$. Then, $p>1$ and $d \neq 0$. Consider the three cases for $n=2,3,4$.

Case 1. If $n=2$, then, $\zeta$ have two nodes $a_{1}$ and $a_{2}$. So, $G^{*}$ is 1-regular, and $a_{1}$ and $a_{2}$ have the same AS $A S\left(a_{1}\right)=$ $\left(t_{N}\left(a_{1} a_{2}\right), f_{N}\left(a_{1} a_{2}\right)\right)$.

Case 2. If $n=3$, then there are three nodes $a_{1}, a_{2}, a_{3}$ with edges $a_{1} a_{2}, a_{2} a_{3}, a_{1} a_{3}$ in $\zeta$ (see Figure 4). Let the edge-MV $a_{1} a_{2}, a_{2} a_{3}$, and $a_{1} a_{3}$ be $\left(t_{N}\left(a_{1} a_{2}\right), f_{N}\left(a_{1} a_{2}\right)\right),\left(t_{N}\left(a_{2} a_{3}\right)\right.$, $\left.f_{N}\left(a_{2} a_{3}\right)\right)$, and $\left(t_{N}\left(a_{1} a_{3}\right), f_{N}\left(a_{1} a_{3}\right)\right)$, respectively.

Since $\zeta$ is $d=\left(d_{1}, d_{2}\right)-\mathrm{RVG}, d\left(a_{i}\right)=\left(d_{1}, d_{2}\right)$, for $i=$ $1,2,3$. Hence, we have

$$
\begin{gathered}
t_{N}\left(a_{1} a_{2}\right)+t_{N}\left(a_{2} a_{3}\right)=d_{1}, \\
t_{N}\left(a_{2} a_{3}\right)+t_{N}\left(a_{1} a_{3}\right)=d_{1}, \\
t_{N}\left(a_{1} a_{2}\right)+t_{N}\left(a_{1} a_{3}\right)=d_{1}, \\
f_{N}\left(a_{1} a_{2}\right)+f_{N}\left(a_{2} a_{3}\right)=d_{2}, \\
f_{N}\left(a_{2} a_{3}\right)+f_{N}\left(a_{1} a_{3}\right)=d_{2}, \\
f_{N}\left(a_{1} a_{2}\right)+f_{N}\left(a_{1} a_{3}\right)=d_{2} .
\end{gathered}
$$

Simplifying the above equations, we have

$$
\begin{aligned}
& t_{N}\left(a_{1} a_{2}\right)=t_{N}\left(a_{2} a_{3}\right)=t_{N}\left(a_{1} a_{3}\right)=\frac{d_{1}}{2}, \\
& f_{N}\left(a_{1} a_{2}\right)=f_{N}\left(a_{2} a_{3}\right)=f_{N}\left(a_{1} a_{3}\right)=\frac{d_{2}}{2} .
\end{aligned}
$$

So, each node has the similar AS, i.e., $\operatorname{AS}\left(a_{i}\right)=\left(\left(d_{1} / 2, d_{1} / 2\right),\left(d_{2} / 2, d_{2} / 2\right)\right)$, for $i=1,2,3$ and $G^{*}$ is 2-RG. Here, $G^{*}$ is a cycle and each edge has the MV $\left(d_{1} / 2, d_{2} / 2\right)$.

Case 3. If $n=4$, then, there are four nodes $a_{1}, a_{2}, a_{3}, a_{4}$ of $\zeta$ with edges $a_{1} a_{2}, a_{1} a_{4}, a_{1} a_{3}, a_{2} a_{3}, a_{2} a_{4}, a_{3} a_{4}$ (see Figure 5). Suppose the MV of the edges is $\left(t_{N}\left(a_{1} a_{2}\right), f_{N}\left(a_{1} a_{2}\right)\right)$, $\left(t_{N}\left(a_{1} a_{4}\right), f_{N}\left(a_{1} a_{4}\right)\right), \quad\left(t_{N}\left(a_{1} a_{3}\right), f_{N}\left(a_{1} a_{3}\right)\right), \quad\left(t_{N}\left(a_{2} a_{3}\right)\right.$, $\left.f_{B}\left(a_{2} a_{3}\right)\right), \quad\left(t_{N}\left(a_{2} a_{4}\right), f_{N}\left(a_{2} a_{4}\right)\right), \quad$ and $\quad\left(t_{N}\left(a_{3} a_{4}\right)\right.$, $\left.f_{N}\left(a_{3} a_{4}\right)\right)$, respectively. Since $\zeta$ is $\left(d_{1}, d_{2}\right)$-RVG, $d\left(a_{i}\right)=$ $\left(d_{1}, d_{2}\right)$, for $i=1,2,3,4$. Hence, we have

$$
\begin{aligned}
t_{N}\left(a_{1} a_{2}\right)+t_{N}\left(a_{1} a_{3}\right)+t_{N}\left(a_{1} a_{4}\right) & =d_{1}, \\
t_{N}\left(a_{2} a_{1}\right)+t_{N}\left(a_{2} a_{3}\right)+t_{N}\left(a_{2} a_{4}\right) & =d_{1}, \\
t_{N}\left(a_{3} a_{1}\right)+t_{N}\left(a_{3} a_{2}\right)+t_{N}\left(a_{3} a_{4}\right) & =d_{1}, \\
t_{N}\left(a_{4} a_{1}\right)+t_{N}\left(a_{4} a_{2}\right)+t_{N}\left(a_{4} a_{3}\right) & =d_{1}, \\
f_{N}\left(a_{1} a_{2}\right)+f_{N}\left(a_{1} a_{3}\right)+f_{N}\left(a_{1} a_{4}\right) & =d_{2}, \\
f_{N}\left(a_{2} a_{1}\right)+f_{N}\left(a_{2} a_{3}\right)+f_{N}\left(a_{2} a_{4}\right) & =d_{2}, \\
f_{N}\left(a_{3} a_{1}\right)+f_{N}\left(a_{3} a_{2}\right)+f_{N}\left(a_{3} a_{4}\right) & =d_{2}, \\
f_{N}\left(a_{4} a_{1}\right)+f_{N}\left(a_{4} a_{2}\right)+f_{N}\left(a_{4} a_{3}\right) & =d_{2} .
\end{aligned}
$$

On simplification, we obtain 


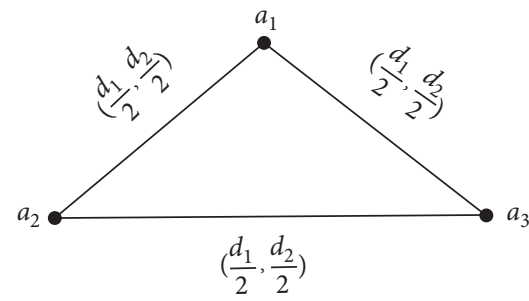

FIgURE 4: $\zeta$ is a $\left(d_{1}, d_{2}\right)$-regular vague graph with $G^{*}$ cyclic and 2 RG.

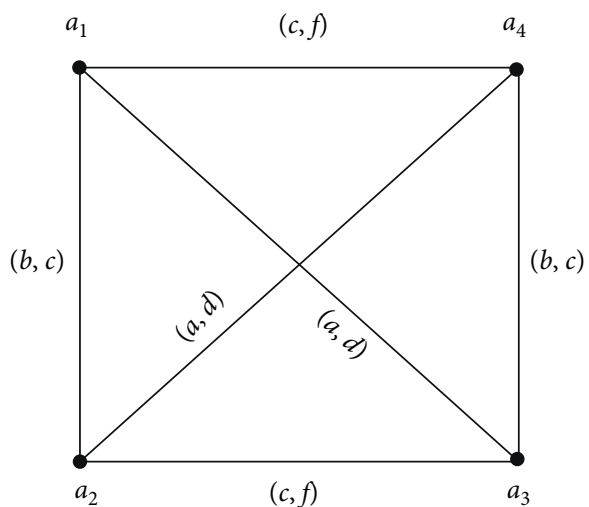

FIGURE 5: $\zeta$ is a $\left(d_{1}, d_{2}\right)$-regular vague graph with $G^{*} 3$-RG or $2-\mathrm{RG}$ or 1-RG.

$$
\begin{aligned}
t_{N}\left(a_{1} a_{3}\right) & =t_{N}\left(a_{2} a_{4}\right), t_{N}\left(a_{1} a_{2}\right)=t_{N}\left(a_{3} a_{4}\right), t_{N}\left(a_{1} a_{4}\right) \\
& =t_{B}\left(a_{2} a_{3}\right), \\
f_{N}\left(a_{1} a_{3}\right) & =f_{N}\left(a_{2} a_{4}\right), f_{N}\left(a_{1} a_{2}\right)=f_{N}\left(a_{3} a_{4}\right), f_{N}\left(a_{1} a_{4}\right) \\
& =f_{N}\left(a_{2} a_{3}\right) .
\end{aligned}
$$

Let $t_{N}\left(a_{1} a_{3}\right)=t_{N}\left(a_{2} a_{4}\right)=a, t_{N}\left(a_{1} a_{2}\right)=t_{N}\left(a_{3} a_{4}\right)=b$, $t_{N}\left(a_{1} a_{4}\right)=t_{N}\left(a_{2} a_{3}\right)=c$, and $f_{N}\left(a_{1} a_{3}\right)=f_{N}\left(a_{2} a_{4}\right)=d$, $f_{N}\left(a_{1} a_{2}\right)=f_{N}\left(a_{3} a_{4}\right)=e$, and $f_{N}\left(a_{1} a_{4}\right)=f_{N}\left(a_{2} a_{3}\right)=f$.

Since that $E \neq \varnothing$, at least one of the edges-MV should be nonzero. If all the edges-MVs are non-zero, then $G^{*}$ is 3-RG and every node of AS has the elements $((a, b, c),(d, e, f))$ in ascending order.

If any two edges-MVs are nonzero, then $G^{*}$ is $2-\mathrm{RG}$ and each node AS has those two nonzero MVs in the ascending order. If one edge-MVs is nonzero, then $G^{*}$ is $1-\mathrm{RG}$ and each node AS has that nonzero MV.

Theorem 2. Let $\zeta=(M, N)$ be a $\left(d_{1}, d_{2}\right)-R V G$ of the q-regular $C G G^{*}$ and every node has the same AS $\left(\left(r_{1}, r_{2}, \ldots, r_{q}\right),\left(s_{1}, s_{2}, \ldots, s_{q}\right)\right), r_{i} \in[0,1]$ and $s_{i} \in[0,1]$, $i=1,2, \ldots, q$. Then, the $V L G L(\zeta)$ of $\zeta$ is regular iff either $N$ is constant or $N$ has exactly three values so that $r_{1}=r_{2}=\cdots=r_{q-1}, \quad r_{i}<r_{q} \leq 1$, for $i=1,2, \ldots, q-1$ and $s_{2}=s_{3}=\cdots=s_{q-1}=s_{q}, 0 \leq s_{1}<s_{i}$, for $i=2, \ldots, q-1$.

Proof. Let the VLG $L(\zeta)$ of $\zeta$ be $\left(t_{1}, t_{2}\right)$-regular. Let $N(e)=$ $\left(r_{i}, s_{i}\right)$ for some $i=1,2, \ldots, q$, where $e=a b$ be any edge of $\zeta$.
Then, every $a$ and $b$ is neighbor with $(q-1)$ edges with MVs $\left(r_{1}, s_{1}\right),\left(r_{2}, s_{2}\right), \ldots,\left(r_{i-1}, s_{i-1}\right),\left(r_{i+1}, s_{i+1}\right), \ldots,\left(r_{q}, s_{q}\right)$. So, the node $e$ in $L(\zeta)$ is neighbor with $2(q-1)$ edges with MVs $\left(r_{1} \wedge r_{i}, s_{1} \vee s_{i}\right),\left(r_{2} \wedge r_{i}, s_{2} \vee s_{i}\right), \quad \ldots,\left(r_{i-1} \wedge r_{i}, s_{i-1} \vee s_{i}\right)$, $\left(r_{i+1} \wedge r_{i}, s_{i+1} \vee s_{i}\right), \ldots,\left(r_{q} \wedge r_{i}, s_{q} \vee s_{i}\right)$, each appearing twice. So, $d_{L(\zeta)}(e)=\left(d_{L(\zeta)}^{t}(e), d_{L(\zeta)}^{f}(e)\right)$, where

$$
\begin{aligned}
d_{L(\zeta)}^{t}(e) & =2 \sum_{j \neq i} r_{j} \wedge r_{i} \\
& =2 \sum_{j=1}^{i-1} r_{j} \wedge r_{i}+2 \sum_{j=i+1}^{q} r_{j} \wedge r_{i} \\
& =2 \sum_{j=1}^{i-1} r_{j}+2 \sum_{j=i+1}^{q} r_{i}, \quad\left(\text { since } r_{1} \leq r_{2} \leq \cdots \leq r_{q}\right) \\
& =2 \sum_{j=1}^{i-1} r_{j}+2(q-i) r_{i},
\end{aligned}
$$

and

$$
\begin{aligned}
d_{L(\zeta)}^{f}(e) & =2 \sum_{j \neq i} s_{j} \vee s_{i} \\
& =2 \sum_{j=1}^{i-1} s_{j} \vee s_{i}+2 \sum_{j=i+1}^{q} s_{j} \vee s_{i} \\
& =2 \sum_{j=1}^{i-1} s_{i}+2 \sum_{j=i+1}^{q} s_{j}, \quad\left(\text { since } s_{1} \leq s_{2} \leq \cdots \leq s_{q}\right) \\
& =2(i-1) s_{i}+2 \sum_{j=i+1}^{q} s_{j} .
\end{aligned}
$$

This holds for every edge $e$ of $\zeta$. Since $L(\zeta)$ is $\left(t_{1}, t_{2}\right)$-regular, $d_{L(\zeta)}(e)=\left(t_{1}, t_{2}\right)$ for each node set $e$ in $L(\zeta)$. Thus, $2 \sum_{j=1}^{i-1} r_{j}+2(q-i) r_{i}=t_{1}$ and $2(i-1) s_{i}+$ $2 \sum_{j=i+1}^{q} s_{j}=t_{2}$, i.e.,

$$
\begin{aligned}
& \sum_{j=1}^{i-1} r_{j}+(q-i) r_{i}=\frac{t_{1}}{2} \\
& (i-1) s_{i}+\sum_{j=i+1}^{q} s_{j}=\frac{t_{2}}{2}, \quad \text { for all } i=1,2, \ldots, q .
\end{aligned}
$$

By equation (13), we have

$$
\begin{array}{r}
(q-1) r_{1}=\frac{t_{1}}{2} \\
\sum_{j=2}^{q} s_{j}=\frac{t_{2}}{2}, \\
r_{1}+(q-2) r_{2}=\frac{t_{1}}{2} \\
s_{2}+\sum_{j=3}^{q} s_{j}=\frac{t_{2}}{2},
\end{array}
$$




$$
\begin{aligned}
r_{1}+r_{2}+(q-3) r_{3} & =\frac{t_{1}}{2} \\
2 s_{3}+\sum_{j=4}^{q} s_{j} & =\frac{t_{2}}{2}, \\
r_{1}+r_{2}+\cdots+r_{q-2}+r_{q-1} & =\frac{t_{1}}{2} \\
(q-2) s_{q-1}+s_{q} & =\frac{t_{2}}{2}, \\
\left(r_{1}+r_{2}+\cdots+r_{q-1}\right) & =\frac{t_{1}}{2} \\
(q-1) s_{q} & =\frac{t_{2}}{2} .
\end{aligned}
$$

Simplifying equations (13)-(18), we have the following:

$$
\begin{aligned}
& r_{1}=r_{2}=\cdots=r_{q} \\
& s_{2}=s_{3}=s_{q-1}=s_{q} .
\end{aligned}
$$

If $r_{1}=r_{q}$ and $s_{1}=s_{q}$, then $N$ is constant; otherwise, $N$ has three values so that $r_{1}=r_{2}=\cdots=r_{q-1}$ and $s_{2}=s_{3}=$ $\cdots=s_{q-1}=s_{q}$.

On the contrary, suppose $N$ is constant or $N$ have just three values so that $r_{1}=r_{2}=\cdots=r_{q-1}, \quad r_{i}<r_{q} \leq 1$, for $i=1,2, \ldots, q-1$, and $s_{2}=s_{3}=\cdots=s_{q-1}=s_{q}, 0 \leq s_{1}<s_{i}$, for $i=2, \ldots, q-1$. If $N$ is constant, let $N(a b)=\left(k_{1}, k_{2}\right)$, $\forall a b \in E$. Then, $k_{1}=r_{i}$ and $k_{2}=s_{i}, \forall i=1,2, \ldots, q$. So, $r_{j} \wedge r_{i}=k_{1}$ and $s_{j} \vee s_{i}=k_{2}, \forall j \neq i$. Otherwise, suppose $r_{j}=k_{1}, \forall j=1,2, \ldots, q-1$ and $s_{j}=k_{2}, \forall j=2, \ldots, q$. Next, $r_{j}=k_{1}<r_{q}$ and $s_{1}<s_{j}=k_{2}$.

So, $r_{j} \wedge r_{i}=k_{1}$ and $s_{j} \vee s_{i}=k_{2}, \forall j \neq i$. Therefore, in all the cases, $r_{j} \wedge r_{i}=k_{1}$ and $s_{j} \vee s_{i}=k_{2}, \forall j \neq i$. Thus, for any node $e=a b \in L(\zeta)$, we have

$$
\begin{aligned}
d_{L(\zeta)}(e) & =\left(d_{L(\zeta)}^{t}(e), d_{L(\zeta)}^{f}(e)\right) \\
& =\left(2 \sum_{j \neq i} r_{j} \wedge r_{i}, 2 \sum_{j \neq i} s_{j} \vee s_{i}\right) \\
& =\left(2 \sum_{j \neq i} k_{1}, 2 \sum_{j \neq i} k_{2}\right) \\
& =\left(2(q-1) k_{1}, 2(q-1) k_{2}\right) .
\end{aligned}
$$

Hence, $L(\zeta)$ is a $\left(2(q-1) k_{1}, 2(q-1) k_{2}\right)$-regular.

Remark 4. The RVG complement does not have to be regular. This can be observed in the following example.

Example 4. Consider the VG $\zeta$ as Figure 6.

Here, $\operatorname{deg}(a)=\operatorname{deg}(b)=\operatorname{deg}(c)=(0.2,1.2)$. So, $\zeta$ is $(0.2,1.2)$-regular. But in its complement $\bar{\zeta}, \operatorname{deg}(a)=$ $(0.6,0.4), \operatorname{deg}(b)=(0.4,0.5), \operatorname{deg}(c)=(0.6,0.5)$.

So, $\bar{\zeta}$ is not regular.

\section{Vague Detour $g$-Distance and Vague Detour $g$-Periphery}

Definition 11. The length of a $x-y$ strong path $p$ between $x$ and $y$ in a $\operatorname{CVG} \zeta=(M, N)$ is said to be a vague detour (VD) $g$-distance if there is no other strong path longer than $p$ between $x$ and $y$, and we show this by $V D_{g}(x, y)$. Any $x-y$ strong path whose length is $V D_{g}(x, y)$ is named a $x-y$ vague $g$-detour.

Example 5. Let $\zeta=(M, N)$ be a CVG of the graph $G^{*}$, where $V=\{x, y, z, w, k\} \quad$ and $E=\{(x, y),(z, w)$, $(y, z),(x, w),(x, z),(x, k),(k, w)\}$. For the VG $\zeta$ in Figure 7 , it is seen that all arcs except $(k, w)$ and $(y, w)$ are strong arcs and the VD $g$-distance of two nodes are given as follows:

$$
\begin{gathered}
V D_{g}(x, y)=1, \\
V D_{g}(y, k)=1, \\
V D_{g}(w, k)=4, \\
V D_{g}(z, w)=2, \\
V D_{g}(x, z)=2, \\
V D_{g}(x, w)=2, \\
V D_{g}(y, w)=3, \\
V D_{g}(k, z)=4, \\
V D_{g}(y, z)=3, \\
V D_{g}(k, x)=2 .
\end{gathered}
$$

Definition 12. The length of any smallest strong path from $x$ to $y$ is named the vague geodesic distance, described by $V d_{g}(x, y)$.

The VD $g$-eccentricity $e_{V D}(a)$ for a node $a$ is $\max \left(V \cdot D_{g}(a, x)\right), \forall x \in \zeta$. The set of all VD $g$-eccentricity nodes of $a$, described by $a_{V \cdot D}^{*}$. The VD $g$-radius of $\zeta$, denoted by $\operatorname{rad}_{V \cdot D_{g}}(\zeta)$, is defined as min $\left(e_{V \cdot D_{q}}(x)\right), \forall x \in \zeta$. If $e_{V \cdot D_{g}}(a)=\operatorname{rad}_{V \cdot D_{g}}(\zeta)$, then the node $a$ is the VD $g$-central node of $\zeta$. The $\mathrm{VD} g$-diameter of $\zeta$, denoted by $\operatorname{diam}_{V \cdot D_{g}}(\zeta)$, is defined as $\max \left(e_{V \cdot D_{g}}(x)\right), \quad \forall x \in \zeta$. If $e_{V \cdot D_{g}}(a)=\operatorname{diam}_{V \cdot D_{g}}(\zeta)$, then the node $a$ is named the VD $g$-peripheral node of $\zeta$.

Example 6. For the connected-VG $\zeta$ in Figure 7, $e_{V \cdot D_{g}}(x)=2, \quad e_{V \cdot D_{g}}(y)=3, \quad e_{V \cdot D_{g}}(k)=4, \quad e_{V \cdot D_{g}}(w)=4$, $e_{V \cdot D_{g}}(z)=4$, and $\operatorname{rad}_{V \cdot D_{g}}(\zeta)=1, \operatorname{diam}_{V \cdot D_{g}}(\zeta)=4$.

Definition 13. The vague subgraph of the $\operatorname{VG} \zeta=(M, N)$, whose nodes are only the VD $g$-peripheral nodes is named a VD $g$-periphery of $\zeta$, and it is denoted by $\operatorname{Per}_{V \cdot D_{g}}(\zeta)$.

Definition 14. If every node of a CVG $\zeta=(M, N)$ is a VD $g$-eccentric node, then $\zeta$ is said to be a VD $g$-eccentric vague 


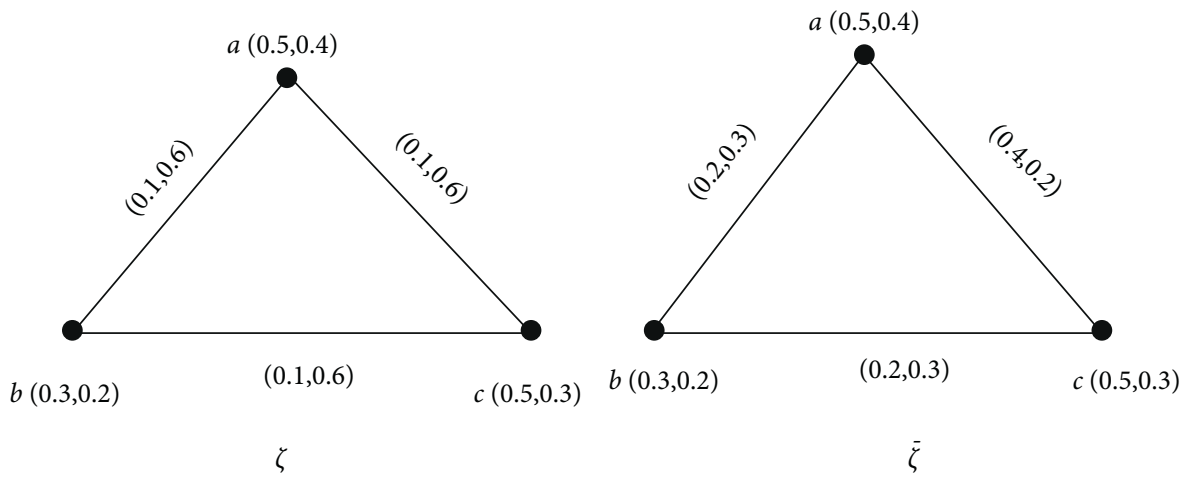

Figure 6: Vague graph $\zeta$ and its complement $(\bar{\zeta})$.

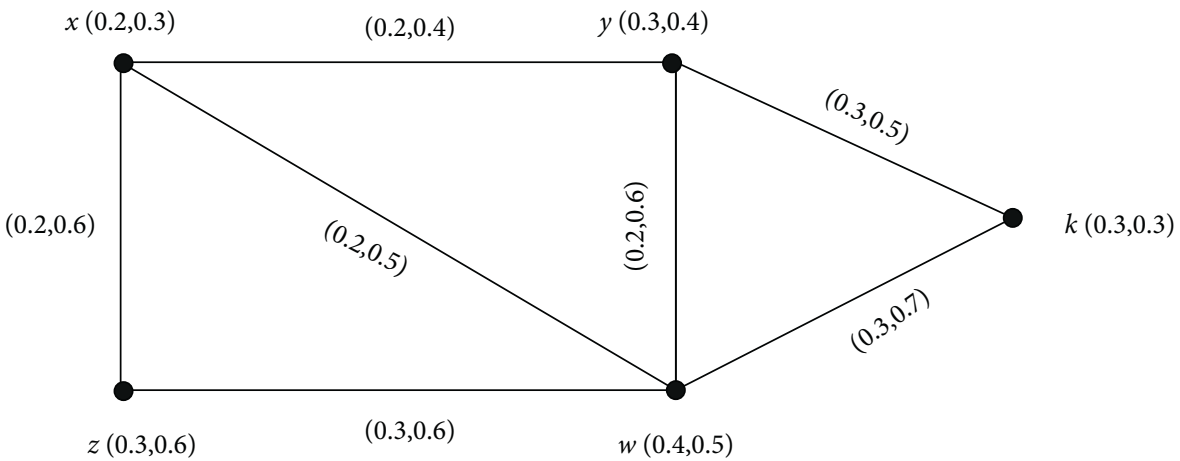

Figure 7: Connected vague graph $\zeta$.

graph. The vague subgraph of $\zeta$ formed by the set of all vague $g$-eccentric nodes of $\zeta$ is named a VD $g$-eccentric vague subgraph of $\zeta$, it is described by $E_{C C_{V \cdot D_{g}}}(\zeta)$

Example 7. For the VG of Figure 8, all nodes $x, y, z$, and $w$ are VD $g$-periphery nodes since $e_{V \cdot D_{g}}(x)=e_{V \cdot D_{g}}(y)=$ $e_{V \cdot D_{g}}(z)=e_{V \cdot D_{g}}(w)=2, \operatorname{diam}_{V \cdot D_{g}}(\zeta)=2$. Also, we have

$$
\begin{aligned}
& x_{V \cdot D_{g}}^{*}=\{y\}, \\
& y_{V \cdot D_{g}}^{*}=\{x\}, \\
& z_{V \cdot D_{g}}^{*}=\{w\}, \\
& w_{V \cdot D_{g}}^{*}=\{z\} .
\end{aligned}
$$

Theorem 3. A VG $\zeta$ is a VD g-self-centered if and only if each node of $\zeta$ is a VD g-eccentric.

Proof. Assume $\zeta$ is a VD $g$-self centered graph and let $y$ be a node in $\zeta$. Let $x \in y_{V \cdot D_{g}}^{*}$. So, $e_{V \cdot D_{g}}(y)=V \cdot D_{g}(x, y)$. Since $\zeta$ is a VD $g$-self centered vague graph, $e_{V \cdot D_{g}}(x)=$ $e_{V \cdot D_{g}}(y)=V \cdot D_{g}(x, y)$, and this implies that $y \in x_{V \cdot D_{g}}^{*}$. So, $y$ is a VD $g$-eccentric vertex of $\zeta$. Conversely, let each vertex of $\zeta$ is a VD $g$-eccentric node. If possible, suppose $\zeta$ be not VD $g$-self-centered vague graph. Then, $\operatorname{rad}_{V \cdot D_{g}}(\zeta) \neq$ $\operatorname{diam}_{V \cdot D_{g}}(\zeta)$, and there exists a node $s \in \zeta$ so that $e_{V \cdot D_{g}}(s)=\operatorname{diam}_{V \cdot D_{g}}(\zeta)$. Also, let $p \in s_{V \cdot D_{g}}^{*}$ Let $u$ be a $s-p$ $\mathrm{VD}$ in $\zeta$. So, there must have a node $q$ on $u$ for which the

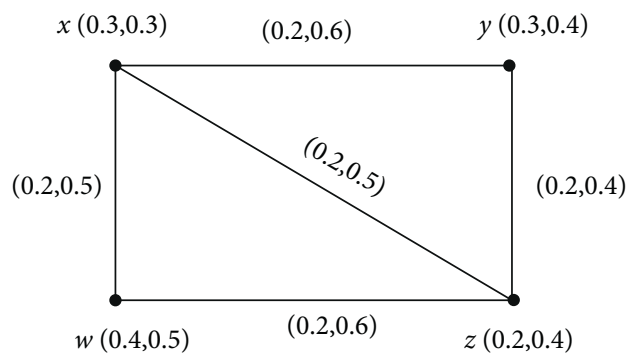

Figure 8: Connected vague graph $\zeta$.

node $q$ is not a VD $g$-eccentric node of $u$. Also, $q$ cannot be a VD $g$-eccentric node of each other node. Again, if $q$ be a VD $g$-eccentric node of a node $x$ (say), it means $q \in x_{V \cdot D}^{*}$. Then, there exists an extension of a $x-q$ vague $g$-detour up to $s$ or up to $p$. But this contradicts the facts that $q \in x_{V \cdot D_{q}}^{*}$. Therefore, $\operatorname{rad}_{V \cdot D_{g}}(\zeta)=\operatorname{diam}_{V \cdot D_{g}}(\zeta)$ and $\zeta$ is a VD $g$-self centered vague graph.

Theorem 4. If $\zeta$ is a VD g-self-centered graph, then $\operatorname{rad}_{V \cdot D_{g}}(\zeta)=$ diam $_{V \cdot D_{g}}(\zeta)=n-1$, where $n$ is the number of nodes of $\zeta$.

Proof. Let $\zeta$ be a VD $g$-self-centered graph. If possible, let $\operatorname{diam}_{V \cdot D_{g}}(\zeta)=l<n-1$. Let $u_{1}$ and $u_{2}$ be two distinct VD $g$-peripheral path. Let $r \in u_{1}, s \in u_{2}$. So, there exists a strong path between $r$ and $s$ because of connectedness of $\zeta$. Then, there exists nodes on $u_{1}$ and $u_{2}$, whose eccentricity $>l$, but 
this is impossible because $\operatorname{diam}_{V \cdot D_{g}}(\zeta)=l$. Hence, $u_{1}$ and $u_{2}$ are not distinct. Since $u_{1}$ and $u_{2}$ are arbitrary, so there exists a node $t$ in $\zeta$ such that $t$ is a common in all VD peripheral paths. So, $e_{V \cdot D}(t)<l$, which is impossible, because $\zeta$ is a VD $g$-self-centered. Therefore, $\quad \operatorname{diam}_{V \cdot D_{g}}(\zeta)=n-1=$ $\operatorname{rad}_{V \cdot D_{g}}(\zeta)$

Corollary 1. For a CVG $\zeta, \operatorname{per}_{V \cdot D_{g}}(\zeta)=\zeta$ if and only if the VD g-eccentricity of every node of $\zeta$ is $n-1$, $n=$ number of no de $\sin \zeta$.

Proof. Let $\operatorname{Per}_{V \cdot D_{g}}(\zeta)=\zeta$. Then, $e_{V \cdot D_{g}}(p)=\operatorname{diam}_{V \cdot D_{g}}(\zeta)$, $\forall p \in \zeta$. So, each node of $\zeta$ is a VD $g$-periphery node of $\zeta$. So, $\zeta$ is a self-centered vague graph and $\operatorname{diam}_{V \cdot D_{g}}(\zeta)=$ $\operatorname{rad}_{V \cdot D_{g}}(\zeta)=n-1$. Therefore, the VD $g$-eccentricity of every node of $\zeta$ is $n-1$.

Conversely, let the VD $g$-eccentricity of every node of $\zeta$ is $n-1$. So, diam ${ }_{V \cdot D_{g}}(\zeta)=\operatorname{rad}_{V \cdot D_{g}}(\zeta)=n-1$. All nodes of $\zeta$ are VD $g$-peripheral nodes, and hence $\operatorname{Per}_{V \cdot D_{g}}(\zeta)=\zeta$.

Corollary 2. For a $C V G \zeta, E_{C C_{V \cdot D g}}(\zeta)=\zeta$ if and only if the VD g-eccentricity of every node of $\zeta$ is $n-1, n=$ number of no de sin $\zeta$.

Proof. Let $E_{C C_{V \cdot D_{g}}}(\zeta)=\zeta$. So, all nodes of $\zeta$ are VD $g$-eccentric node. Hence, $\zeta$ is self-centered vague graph and $\operatorname{diam}_{V \cdot D_{g}}(\zeta)=n-1$. So, the VD $g$-eccentricity of every node of $\zeta$ is $n-1$.

Conversely, let the VD $g$-eccentricity of every node of $\zeta$ is $n-1$. So, $\operatorname{rad}_{V \cdot D_{g}}(\zeta)=\operatorname{diam}_{V \cdot D_{g}}(\zeta)=n-1$. Therefore, all nodes of $\zeta$ are VD $g$-peripheral nodes as well as VD g-eccentric node. So, $E_{C C_{V \cdot D_{g}}}(\zeta)=\zeta$.

Theorem 5. In a CVG $\zeta$, a node $x$ is a VD g-eccentric node if and only if $x$ is a VD $g$-peripheral node.

Proof. Let $x$ be a VD $g$-eccentric node of $\zeta$ and let $x \in y_{V \cdot D_{g}}^{*}$. Let $z$ and $w$ be two VD $g$-peripheral nodes, then $V \cdot D_{g}(z, w)=\operatorname{diam}_{V \cdot D_{g}}(\zeta)=k$ (say). Let $p_{1}$ and $p_{2}$ be any $z-w$ and $y-x$ vague $g$-detour in $\zeta$, respectively. There arise two cases:

Case 1: if $x$ is not internal node in $\zeta$, i.e., there is only one node, say $s$ which is adjacent to $x$. So, $s \in P_{2}$. Since $\zeta$ is connected, $s$ is connected to a node of $p_{1}$, say $p^{\prime}$. Therefore, either $s \prime \in p_{2}$ or $s \prime \in\left(p_{1} \cap p_{2}\right)$. Thus, in any case, the path from $y$ to $z$ or $y$ to $w$ through $s$ and $s \prime$ is longer than $p_{2}$. But it is impossible, since $x$ is a VD $g$-eccentric node of $y$. So, $e_{V \cdot D_{g}}(y)=\operatorname{diam}_{V \cdot D_{g}}(\zeta)$, i.e., $b$ is a VD $g$-peripheral node of $\zeta$.

Case 2: if $x$ is an internal node in $\zeta$, then $\exists$ a connection between $x$ to $z$ and $x$ to $w$ because of connectedness of $\zeta$. Then, $y-x$ vague $g$-detour can be extended to $z$ or $w$. This is impossible because $x$ is a VD $g$-eccentric node of $y$. Hence, $e_{V \cdot D_{g}}(y)=\operatorname{diam}_{V \cdot D_{g}}(\zeta)$, i.e., $x$ is a VD $g$-peripheral node of $\zeta$. Conversely, we suppose that $x$ be a VD $g$-peripheral node of $\zeta$. So, $\exists$ a VD $g$-peripheral node, say $y$ (distinct from $x$ ). Therefore, $x$ is a VD $g$-eccentric node of $y$.
Definition 15. In a CVG $\zeta$, a node $y$ is said to be a VD $g$-boundary node of a node $x$, if $V \cdot D_{g}(x, y) \geq V \cdot D_{g}(x, z)$, for each $z$ in $\zeta$, where $z$ is a neighbor of $y$.

The set of all VD $g$-boundary nodes of $x$ described by $x_{V \cdot D_{g}^{\prime}}$.

Example 8. Consider the CVG $\zeta$ in Figure 9.

In this example, we have

$$
\begin{aligned}
& x_{V \cdot D_{g}}^{\prime}=\{t, s\}, \\
& y_{V \cdot D_{g}}^{\prime}=\{x, t, s\}, \\
& z_{V \cdot D_{g}}^{\prime}=\{x, t, s\}, \\
& w_{V \cdot D_{g}}^{\prime}=\{x, t, s\}, \\
& t_{V \cdot D_{g}}^{\prime}=\{x, s\}, \\
& s_{V \cdot D_{g}}^{\prime}=\{x, t\} .
\end{aligned}
$$

Here, $x, t$, and $s$ are the VD $g$-boundary nodes of $\zeta$.

Theorem 6. A CVG $\zeta$ is a vague tree if and only if $\zeta$ is a vague g-detour graph.

Proof. Let $\zeta$ be a vague tree. Then, between any two nodes in $\zeta$, there is exactly one vague strong path. So, $V \cdot D_{g}(x, y)=V \cdot d_{g}(x, y)$, for any two nodes $x, y$ in $\zeta$. Hence, $\zeta$ is a vague $g$-detour graph.

On the contrary, let $\zeta$ be a vague $g$-detour graph, which has $n$ nodes. Then, $V \cdot D_{g}(x, y)=V \cdot d_{g}(x, y)$, for any two nodes $x, y$ in $\zeta$. If $n=z$, then $\zeta$ is a vague tree. Let $n \geq 3$. If possible, let $\zeta$ be not a vague tree. So, there exists two nodes $s, t$ in $\zeta$ for which there is at least two strong paths between $s$ and $t$. Let $Q_{1}$ and $Q_{2}$ be two $s-t$ vague strong paths. So, $Q_{1} \cup Q_{2}$ has a cycle $C$ (say) in $\zeta$. If nodes $x$ and $y$ are adjacent nodes in $\zeta$, then we have $V \cdot d_{g}(x, y)=1$ and $V \cdot D_{g}(x, y)>1$. This contradicts the fact that $V \cdot D_{g}(x, y)=$ $V \cdot d_{g}(x, y)$. Hence, $\zeta$ is a vague tree.

Definition 16. A node $x$ in a VG $\zeta$ is called a $\operatorname{VEN}$ of $\zeta$ if $y$ is the only strong neighbor of $x$, where $y \in \zeta$.

Example 9. For the VG $\zeta$ in Figure 9, the nodes $x, t$, and $s$ are VENs of $\zeta$.

Theorem 7. A node $y$ in a vague tree $\zeta$ is a VD g-boundary node if and only if $y$ is a VEN.

Proof. Let a node $y$ be a VD $g$-boundary node for a node $x$ in a vague tree $\zeta$. Let $E$ be a maximum vague spanning tree in $\zeta$, which is unique in $\zeta$.

\section{Application of Vague Detour $g$-Distance in the Transportation System}

Today, the issue of transportation plays a very important role in human life. If this is done faster and easier, then it can affect the quality of life and human health. Unfortunately, in the past, due to the lack of sufficient vehicles and bad road 


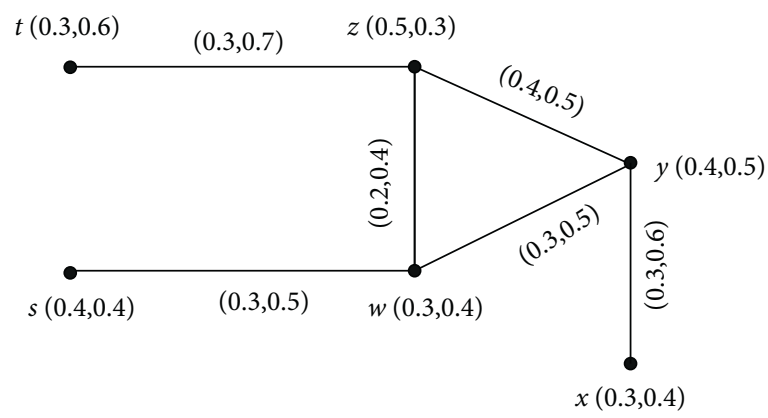

FIgURE 9: Connected vague graph $\zeta$.

TABLE 2: Weight of nodes.

\begin{tabular}{lccccc}
\hline & $A$ & $B$ & $C$ & $D$ & $E$ \\
\hline$t_{A}$ & 0.2 & 0.2 & 0.3 & 0.5 & 0.4 \\
$f_{A}$ & 0.2 & 0.3 & 0.5 & 0.2 & 0.3 \\
\hline
\end{tabular}

TABLE 3: Weight of edges.

\begin{tabular}{lccc}
\hline$\zeta$ & $A-$ Yahya Nejad & $A-$ Beheshti & $A-$ Mehregan \\
\hline$\left(t_{B}, f_{B}\right)$ & $(0.2,0.5)$ & $(0.1,0.8)$ & $(0.2,0.4)$ \\
$\zeta$ & $A-$ Rouhani & Beheshti - Mehregan & Mehregan - Rouhani \\
$\left(t_{B}, f_{B}\right)$ & $(0.2,0.4)$ & $(0.3,0.7)$ & $(0.3,0.7)$ \\
$\zeta$ & Yahya Nejad - Beheshti & & \\
$\left(t_{B}, f_{B}\right)$ & $(0.2,0.7)$ & & \\
\hline
\end{tabular}

conditions, many problems were created for human beings, one of the most important of which is the lack of timely transfer of patients to private hospitals and clinics for treatment. Therefore, in this paper, we intend to express the importance and application of vague detour $g$-distance in the transportation of a patient to the most appropriate hospital in the shortest possible time. For this purpose, we consider four hospitals in Iran (Babol City) named Yahya Nejad (B), Beheshti (C), Mehregan (D), and Rouhani (E), which are shown in the graph with the symbols of $B, C, D$, and $E$. Suppose that a patient lives in location $A$ and must be transported by ambulance to one of these four hospitals in the shortest possible time. In this vague graph, the nodes represent the hospitals and the edges also shows the amount of traffic generated at a certain hour of the day. The weight of the nodes and edges is shown in Tables 2 and 3. The location of hospitals is shown in Figure 10.

The node $D(0.5,0.2)$ shows that this hospital has $50 \%$ of the medical facilities and equipment needed to treat a patient, but does not have $20 \%$ of the necessary tools. The edge $A D$ indicates that $40 \%$ of this route has traffic and congestion caused by vehicles, but $20 \%$ of it is free of cars and vehicles. The vague detour $g$-distance for Figure 11 is as follows:

$$
\begin{gathered}
V D_{g}(A, B)=4, \\
V D_{g}(A, C)=3, \\
V D_{g}(A, D)=3, \\
V D_{g}(A, E)=4, \\
V D_{g}(B, C)=4, \\
V D_{g}(B, D)=3, \\
V D_{g}(B, E)=3, \\
V D_{g}(C, D)=4, \\
V D_{g}(C, E)=3, \\
V D_{g}(D, E)=4 .
\end{gathered}
$$

It is clear that $V D_{g}(A, D)$ has the lowest value, so we conclude that it can be the best choice because, firstly, Mehregan hospital has the most medical equipment and facilities compared to other hospitals, and secondly, A-Mehregan route has the most empty space for patient transfer to medical centers by ambulance. Therefore, governments should provide conditions for patients to be transported to hospitals without stress due to the congestion of roads and intercity traffic, so that they can be treated as soon as possible. 


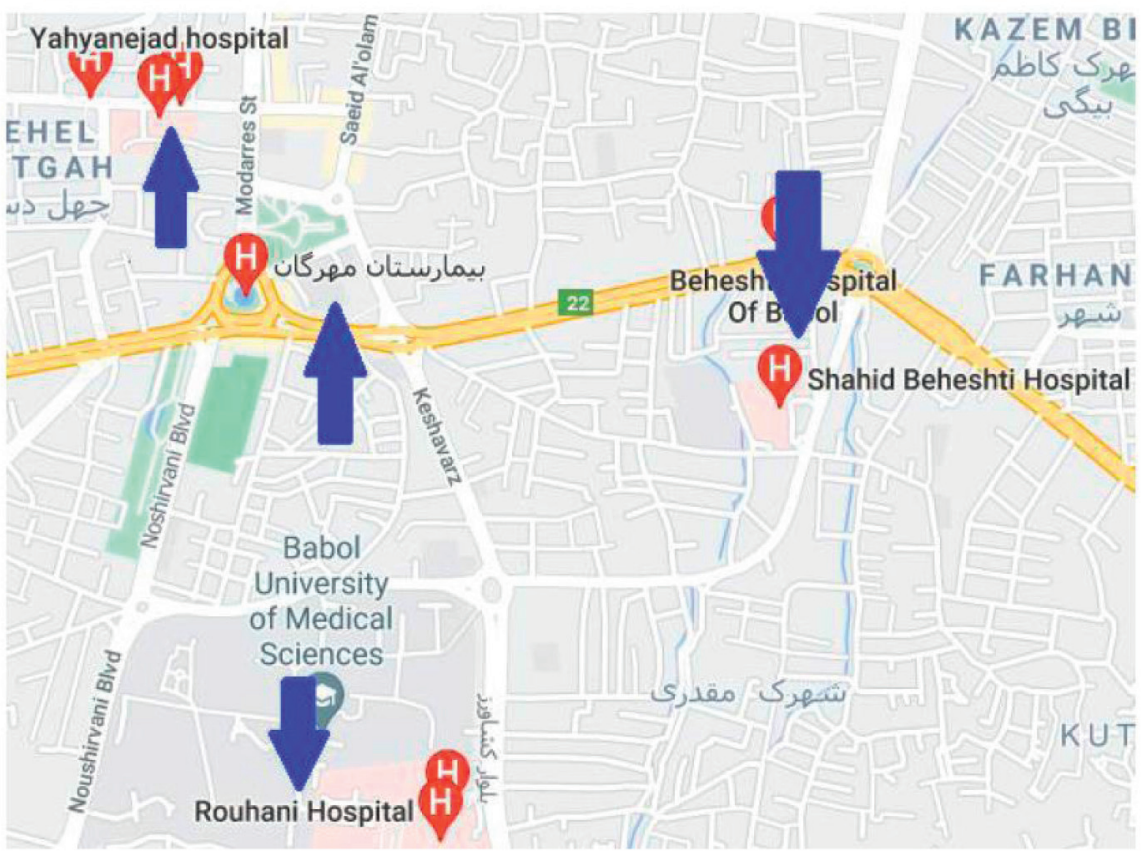

Figure 10: Location of hospitals in Babol City.

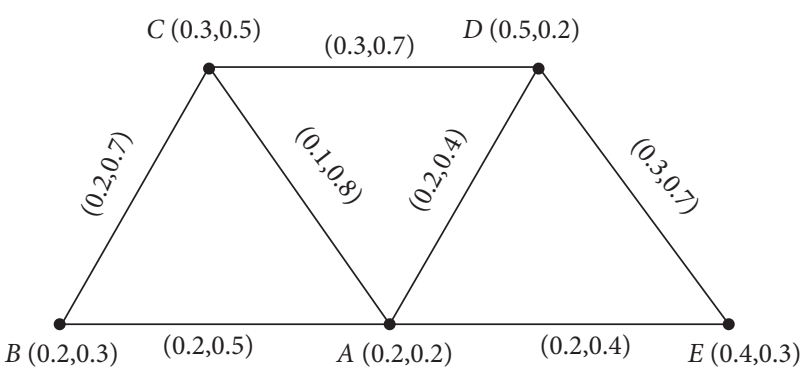

FIgURE 11: Vague graph $\zeta$.

\section{Conclusion}

A VG is an indiscriminately comprehensive structure of an FG that offers higher precision, adaptability, and compatibility to a system when coordinated with systems running on FGs. VGs are so useful tool to examine many issues such as networking, social systems, geometry, biology, clustering, medical science, and traffic plan. Eccentric nodes and $g$-boundary nodes are a practical interest in several areas. In wireless networking, boundary nodes are used to find efficient routes within ad hoc mobile networks. They have also been used in document summarization and in designing secure systems for electrical grids. Hence, in this paper, the adjacency sequence of RVGs is defined with examples. Also, a characterization of vague detour $g$-eccentric node and the concepts of vague detour $g$-boundary nodes, vague detour $g$-interior nodes in a VG are examined. In our future work, we will introduce vague incidence graphs and study the concepts of perfect dominating set, regular perfect dominating set, and independent perfect dominating set on vague incidence graph. Likewise, we will try to define the average connectivity index, parameter and the new concepts of vague connectivity-enhancing node, vague detour g-interior node, and vague detour g-boundary node of a vague incidence tree using maximum vague spanning tree.

\section{Data Availability}

No data were used to support this study.

\section{Conflicts of Interest}

The authors declare no conflicts of interest.

\section{References}

[1] A. Rosenfeld, Fuzzy Graphs, Fuzzy Sets and Their Applications, L. A. Zadeh, K. S. Fu, and M. Shimura, Eds., Academic Press, New York, NY, USA, pp. 77-95, 1975.

[2] L. A. Zadeh, "Fuzzy sets," Information and Control, vol. 8, no. 3, pp. 338-353, 1965.

[3] W.-L. Gau and D. J. Buehrer, "Vague sets," IEEE Transactions on Systems, Man, and Cybernetics, vol. 23, no. 2, pp. 610-614, 1993.

[4] A. Kauffman, "Introduction a la theories des sous-emsembles 503 ous," Masson Cie, vol. 1, pp. 607-618, 1973.

[5] L. A. Zadeh, "Similarity relations and fuzzy orderings," Information Sciences, vol. 3, no. 2, pp. 177-200, 1971.

[6] L. A. Zadeh, "Is there a need for fuzzy logic?" Information Sciences, vol. 178, no. 13, pp. 2751-2779, 2008.

[7] M. Akram, W. A. Dudek, and M. Murtaza Yousof, "Regularity in vague intersection graphs and vague line graphs," Abstract and Applied Analysis, vol. 2014, Article ID 525389, 10 pages, 2014.

[8] M. Akram, A. N. Gani, and A. B. Saeid, "Vague hypergraphs," Journal of Intelligent and Fuzzy Systems, vol. 26, no. 2, pp. 647-653, 2014. 
[9] S. Samanta and M. Pal, "Irregular bipolar fuzzy graphs," International Journal of Fuzzy System Applications, vol. 2, pp. 91-102, 2012.

[10] S. Samanta and M. Pal, "Fuzzy k-competition graphs and p-competition fuzzy graphs," Fuzzy Information and Engineering, vol. 5, no. 2, pp. 191-204, 2013.

[11] A. Nagoorgani and S. R. Latha, "On irregular fuzzy graphs," Applied Mathematical Sciences, vol. 6, pp. 517-523, 2012.

[12] A. Nagoorgani and K. Radha, "On regular fuzzy graphs," Journal of Physical Science, vol. 12, pp. 33-44, 2008.

[13] M. S. Sunitha and S. Mathew, "Fuzzy graph theory: a survey, Ann," Pure and Applied Mathematics Journal, vol. 4, pp. 92-110, 2013.

[14] M. S. Sunitha and A. Vijayakumar, "Complement of a fuzzy graph," Indian Journal of Pure and Applied Mathematics, vol. 33, pp. 1451-1464, 2002.

[15] N. Ramakrishna, "Vague graphs," International Journal of Cognitive Computing, vol. 7, pp. 51-58, 2009.

[16] R. Borzooei and H. Rashmanlou, "Domination in vague graphs and its applications," Journal of Intelligent and Fuzzy Systems, vol. 29, no. 5, pp. 1933-1940, 2015.

[17] R. A. Borzooei and H. Rashmanlou, "Degree of vertices in vague graphs," Journal of applied mathematics \& informatics, vol. 33, no. 5_6, pp. 545-557, 2015.

[18] G. Chatrand, H. Escuadro, and P. Zhang, "Detour distance in graph," Journal of Combinatorial Mathematics and Combinatorial Computing, vol. 53, pp. 75-94, 2005.

[19] G. Chatrand, G. L. Johns, and P. Zhang, "Detour number of a graph," Utilitas Mathematica, vol. 64, pp. 97-113, 2003.

[20] G. Chatrand, D. Erwin, G. L. Johns, and P. Zang, "Boundary vertices in graphs," Discrete Math, vol. 263, no. 1-3, pp. 25-34, 2003.

[21] J. P. Linda and M. S. Sunitha, "Fuzzy detour g-centre in fuzzy graphs, Ann," Fuzzy Math Informa, vol. 7, no. 2, pp. 219-228, 2014.

[22] G. Ghorai, "Characterization of regular bipolar fuzzy graphs," Afrika Matematika, vol. 32, no. 5-6, pp. 1043-1057, 2021.

[23] S. Poulik and G. Ghorai, "Detour g-interior nodes and detour g-boundary nodes in bipolar fuzzy graphs with applications," Hacettepe Journal of Mathematics and Statistics, vol. 49, no. 1, pp. 106-119, 2020.

[24] K. R. Bhutani and A. Rosenfeld, "Strong arcs in fuzzy graphs," Information Sciences, vol. 152, pp. 319-322, 2003.

[25] S. Mathew and M. S. Sunitha, "Types of arcs in a fuzzy graph," Information Sciences, vol. 179, no. 11, pp. 1760-1768, 2009.

[26] J. P. Linda and M. S. Sunitha, "On g-eccentric nodes g-boundary nodes and g-interior nodes of a fuzzy graph," International Journal of Mathematical Sciences \& Applications, vol. 2, no. 2, pp. 697-707, 2012.

[27] S. Banitalebi and R. A. Borzooei, "2-Domination in vague graphs," Algebraic Structures and Their Applications, vol. 8, no. 2, pp. 203-222, 2021.

[28] P. K. Kishore Kumar, Y. Talebi, H. Rashmanlou, A. A. Talebi, and F. Mofidnakhaei, "New concept of cubic graph with application," Journal of Multiple-Valued Logic and Soft Computing, vol. 33, pp. 135-154, 2019.

[29] S. Kosari, Y. Rao, H. Jiang, X. Liu, P. Wu, and Z. Shao, "Vague graph structure with application in medical diagnosis," Symmetry Plus, vol. 12, no. 10, p. 1582, 2020.

[30] K. Radha and N. Kumaravel, "Some properties of edge regular fuzzy graphs," Jamal Academic Research Journal, pp. 121-127, 2014.

[31] H. Rashmanlou, S. Samanta, M. Pal, and R. A. Borzooei, "Intuitionistic fuzzy graphs with categorical properties,"
Fuzzy Information and Engineering, vol. 7, no. 3, pp. 317-334, 2015.

[32] S. Samanta, M. Pal, and A. Pal, "New concepts of fuzzy planar graph," International Journal of Advanced Research in Artificial Intelligence, vol. 3, no. 1, pp. 52-59, 2014.

[33] S. Samanta and M. Pal, "Fuzzy tolerance graphs," International Journal of Latest Trends in Mathematics, vol. 1, no. 2, pp. 57-67, 2011.

[34] S. Sahoo, M. Pal, H. Rashmanlou, and R. A. Borzooei, "Covering and paired domination in intuitionistic fuzzy graphs," Journal of Intelligent and Fuzzy Systems, vol. 33, no. 6, pp. 4007-4015, 2017.

[35] A. A. Talebi, N. Mehdipoor, and H. Rashmanlou, "Some operations on vague graphs," Journal of Advanced Research in Pure Mathematics, vol. 6, no. 1, pp. 61-77, 2014.

[36] S. Zeng, M. Shoaib, S. Ali, Q. Abbas, and M. S. Nadeem, "Complex vague graphs and their application in decisionmaking problems," IEEE Access, vol. 8, pp. 174094-174104, 2020.

[37] S. Zeng, M. Shoaib, S. Ali, F. Smarandache, H. Rashmanlou, and F. Mofidnakhaei, "Certain properties of single-valued neutrosophic graph with application in food and agriculture organization," International Journal of Computational Intelligence Systems, vol. 14, no. 1, pp. 1516-1540, 2021.

[38] S. Zeng, Z. Ali, and T. Mahmood, "Novel complex T-spherical dual hesitant uncertain linguistic muirhead mean operators and their application in decision-making," Computer Modeling in Engineering and Sciences, vol. 129, no. 2, pp. 849-880, 2021.

[39] M. Shoaib, S. Kosari, H. Rashmanlou et al., "Notion of complex pythagorean fuzzy graph with properties and application," Journal of Multiple-Valued Logic and Soft Computing, vol. 34, pp. 553-586, 2020.

[40] W. L. Craine, "Characterizations of fuzzy interval graphs," Fuzzy Sets and Systems, vol. 68, no. 2, pp. 181-193, 1994. 